\title{
Imaging features of parotid gland oncocytoma: a case series study
}

\author{
Kun Lv ${ }^{1,2 \#}$, Xin Cao ${ }^{1,2 \#}$, Daoying Geng ${ }^{1,2}$, Jun Zhang ${ }^{1,2}$ \\ ${ }^{1}$ Department of Radiology, Huashan Hospital, Fudan University, Shanghai, China; ${ }^{2}$ Institute of Functional and Molecular Medical Imaging, Fudan \\ University, Shanghai, China \\ Contributions: (I) Conception and design: D Geng; (II) Administrative support: J Zhang; (III) Provision of study materials or patients: K Lv; (IV) \\ Collection and assembly of data: K Lv, X Cao; (V) Data analysis and interpretation: J Zhang; (VI) Manuscript writing: All authors; (VII) Final \\ approval of manuscript: All authors. \\ "These authors contributed equally to this work. \\ Correspondence to: Daoying Geng. Department of Radiology, Huashan Hospital, Fudan University, 12, Wulumuqi Middle Road, Shanghai 200040, \\ China. Email: gdy_2019@163.com; Jun Zhang. Department of Radiology, Huashan Hospital, Fudan University, 12, Wulumuqi Middle Road, \\ Shanghai 200040, China. Email: zhj81828@163.com.
}

Background: Reports regarding the imaging findings of parotid gland oncocytoma are limited and prospective definitive diagnosis is difficult. This case series is aimed at furthering the knowledge of imaging for parotid gland oncocytoma.

Methods: Seven patients with pathologically confirmed parotid gland oncocytoma were identified between January, 2016 and August, 2020. They were subjected to computed tomography, magnetic resonance imaging or ultrasound. Imaging features of lesions were retrospectively analyzed and documented by a senior radiologist.

Results: Among the 7 recruited parotid gland oncocytoma patients, 4 were males and 3 were females. They were aged between 47 and 87 years with a median age of 63 years. The range of maximum diameter of the mass was about $1.6-4.5 \mathrm{~cm}$, with the median size being $3.5 \mathrm{~cm}$. All cases were unilateral single masses. Three patients had their parotid gland oncocytoma located in the deep lobe of the parotid gland and 4 patients had their parotid gland oncocytoma located in the superficial lobe. Most lesions exhibited a clear boundary and irregular shapes or lobulated, and contain cystic components. Local pain was the most common clinical manifestation. Most of the lesions exhibited a hypoechoic mass on ultrasound, soft tissue density on computed tomography, iso to hypointense signal on T1 weighted imaging, slightly hypersignal on T2 weighted imaging, while contrast-enhanced computed tomography, magnetic resonance imaging, and ultrasound showed avid enhancement.

Conclusions: Parotid gland oncocytomas usually exhibit a clear boundary to surrounding parotid tissue, lobulated or irregular shape and avid enhancement, sometimes accompanied with cystic components. Diagnosis of parotid gland oncocytoma should be considered when these features are encountered in clinical practice.

Keywordsı Oncocytoma; parotid gland; case series; diagnostic imaging; magnetic resonance imaging

Submitted Oct 29, 2020. Accepted for publication Feb 03, 2021.

doi: $10.21037 /$ gs-20-790

View this article at: http://dx.doi.org/10.21037/gs-20-790 


\section{Introduction}

Oncocytoma is also known as eosinophilic adenoma. It is a rare benign epithelial tumor accounting for about $0.05 \%$ of all salivary gland tumors and $1 \%$ of parotid gland tumors. It was first described and named by Jaffe in $1932(1,2)$. Currently, few studies have reported imaging findings of parotid gland oncocytoma (PGO), especially ultrasound (US) findings, and most of the available literature are case reports $(2,3)$. The incidence of PGO is very low. It is easily misdiagnosed as other relatively common tumors of parotid gland, such as pleomorphic adenoma (PA), Warthin tumor, lymphoma, and mucoepidermoid carcinoma. Moreover, there is evidence that some cases of PGO exhibit malignant transformations (4). Preoperative diagnosis of masses is important for surgical planning and prognosis. Computed tomography (CT) and magnetic resonance imaging (MRI) can effectively evaluate the status of masses and facilitate differential diagnosis. The high soft tissue contrast of MRI plays an important role in disease diagnosis. Compared to CT and MRI, US is a low-cost, relatively easy to implement, non-invasive tool that does not induce ionizing radiation damage. Contrast enhanced ultrasound (CEUS) provides real time imaging of microvessels in lesions and the perfusion characteristics of contrast agents. Therefore, Seven PGO patients with relatively complete imaging data were recruited to furthering the knowledge of imaging for PGO.

We present the following article in accordance with the MDAR and AME Case Series reporting checklists (available at http://dx.doi.org/10.21037/gs-20-790).

\section{Methods}

\section{Study participants}

The study was conducted in accordance with the Declaration of Helsinki (as revised in 2013). The study was approved by institutional ethics committee of Huashan Hospital, Fudan University (No. KY2013-332) and informed consent was waived due to the retrospective nature of this study. The inclusion criteria: (I) PGO was confirmed by pathological examination; (II) patients with high quality images; (III) complete clinical data. Exclusion criteria: (I) patients without pathological results; (II) poor image quality. According to these criteria, 7 PGO patients with complete clinical and imaging data were recruited between January, 2016 and August, 2020. Three patients were subjected to contrast enhanced CT scan, 4 patients underwent contrast enhanced MRI scanning, and 4 patients received the conventional US examination and CEUS examination.

\section{Imaging acquisition}

CT scan was performed using the GE Bright Speed 16 spiral CT. Enhanced CT were performed after unenhanced scan. The tube voltage was $120 \mathrm{kv}$, tube current $(320 \mathrm{~mA})$, collimator width $(0.75 \mathrm{~mm})$, scanning pitch (1.0), slice thickness ( $3 \mathrm{~mm}$ ), while slice spacing was $3 \mathrm{~mm}$. The scanning range was from the skull base to the thoracic entrance level. The contrast medium iohexol $80-100 \mathrm{~mL}$ $(1.5 \mathrm{~mL} / \mathrm{kg})$ was injected through the cubital vein at a rate of $3.0 \mathrm{~mL} / \mathrm{s}$ and delayed scanning was performed for $42 \mathrm{~s}$.

MRI examination was conducted using a 3.0T MR scanner (Discovery 750, GE Medical System, Milwaukee, WI) with head and neck array coils using the following parameters: For transverse T1WI, repetition time (TR) $=600 \mathrm{~ms}$, echo time $(\mathrm{TE})=9 \mathrm{~ms}$, slice thickness $=4 \mathrm{~mm}$, field-of-view $(\mathrm{FOV})=220 \mathrm{~mm} \times 220 \mathrm{~mm}$; For transverse T2WI, TR $=4,000 \mathrm{~ms}$, TE $=75 \mathrm{~ms}$, slice thickness $=4 \mathrm{~mm}$, FOV $=220 \mathrm{~mm} \times 220 \mathrm{~mm}$.

Gadolinium-diethylenetriamine penta-acetic acid (GdDTPA) (Hokuriku Seiyaku Co., Ltd, Katsuyama, Japan) at a dose of $0.1 \mathrm{mmol} / \mathrm{kg}$ of body weight was administered intravenously at a rate of $2 \mathrm{~mL} / \mathrm{s}$, followed by a $20 \mathrm{~mL}$ saline flush.

US diagnosis was performed using a Mindray Resona 7 scanner (Mindray Medical Solutions; Shenzhen, China) with a high frequency probe $(11 \mathrm{MHz})$. CEUS was performed after routine US examination. The mechanical index of CEUS was 0.08. 2.4 mL of contrast medium, SonoVue (SonoVue; Bracco SpA, Milan, Italy) was injected into the median cubital vein and flushed with $5 \mathrm{~mL}$ of $0.9 \%$ saline. After injection of contrast medium, the echo and enhancement degree of lesions and surrounding tissues were observed.

\section{Image analysis}

All images were retrospectively analyzed and summarized by senior radiologists. The observed features included lesion density, signal intensity, echogenicity, boundary, shape, enhancement degree after contrast. Compared to the rest of parotid gland, the degree of enhancement on enhanced scan was divided into hypoenhancement, isoenhancement and hyperenhancement. 

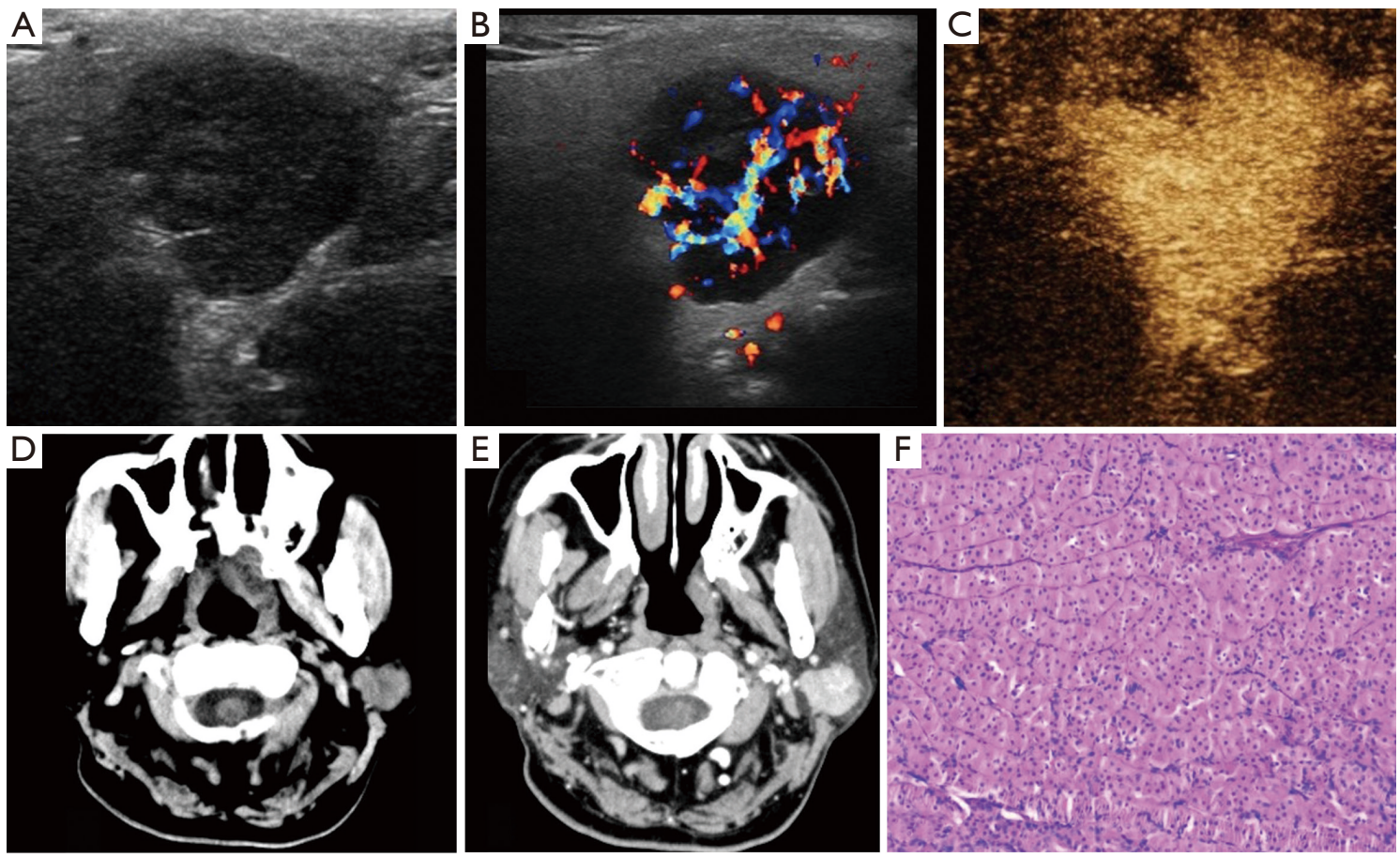

Figure 1 A 55-year-old female with left PGO. (A) Precontrast US showed a hypoechoic mass with clear boundary and an irregular shape; (B) CDFI showed abundant blood flow signal to the mass; (C) after contrast medium injection for $11 \mathrm{~s}$, there was homogeneous hyperenhancement; (D) unenhanced CT scan showed a soft tissue density with a clear boundary and an irregular shape; (E) contrast enhanced CT showed hyperenhancement of the mass; (F) pathological sample showed abundant oncocytes of the mass (hematoxylin and eosin staining, $\times 100$ ). PGO, parotid gland oncocytoma; US, ultrasound; CDFI, color Doppler flow imaging; CT, computed tomography.

\section{Statistical analysis}

The measurement data were calculated by SPSS 22.0 software (IBM Corp., Armonk, NY, USA), such as age and size of lesion. This study is not involving statistical test due to the nature of an observational cases series study.

\section{Results}

Seven PGO patients were recruited in this study, included 4 males and 3 females. The age of patients ranged between 47 and 87 years with a median age of 63 years. The diameters of the tumors ranged between 1.6 and 4.5 $\mathrm{cm}$ with a median size of $3.5 \mathrm{~cm}$. Six patients exhibited a palpable mass, 3 presented with local pain while 1 case was founded by physical examination. All seven cases had unilateral single masses with 3 cases were located in the deep lobe of the parotid gland, and 4 cases were located in the superficial lobe of the parotid gland. The lesions presented with a clear boundary $(6 / 7,85.7 \%)$, irregular shapes or lobulated $(5 / 7,71.4 \%)$, and some had cystic changes $(4 / 7,57.1 \%)$. On ultrasound, 4 patients had a hypoechoic mass and two of them showed hypervascular on color Doppler flow imaging (CDFI). On CEUS, 3 cases showed hyperenhancement, one exhibited hypoenhancement, and in one of them, the size of mass was larger on postcontrast US than precontrast US. Three cases displayed soft tissue density on CT scan, which showed hyperenhancement after contrast enhanced scan (Figure 1). Four cases showed iso/hypo-signals on T1WI, 1 case had slightly hyposignal on T2WI, 1 case showed a slight hypersignal on T2WI, 2 cases showed mixed signals due to cystic components, 3 cases showed hyperenhancement (Figure 2), and 1 case showed hypoenhancement. There was no enhancement on enhanced US/CT/MRI in cystic components, and no obvious calcification was observed in any of the lesions. Three patients underwent biopsy and 4 patients received surgery to obtain pathological samples. The clinical data and imaging features are summarized in Table 1. 

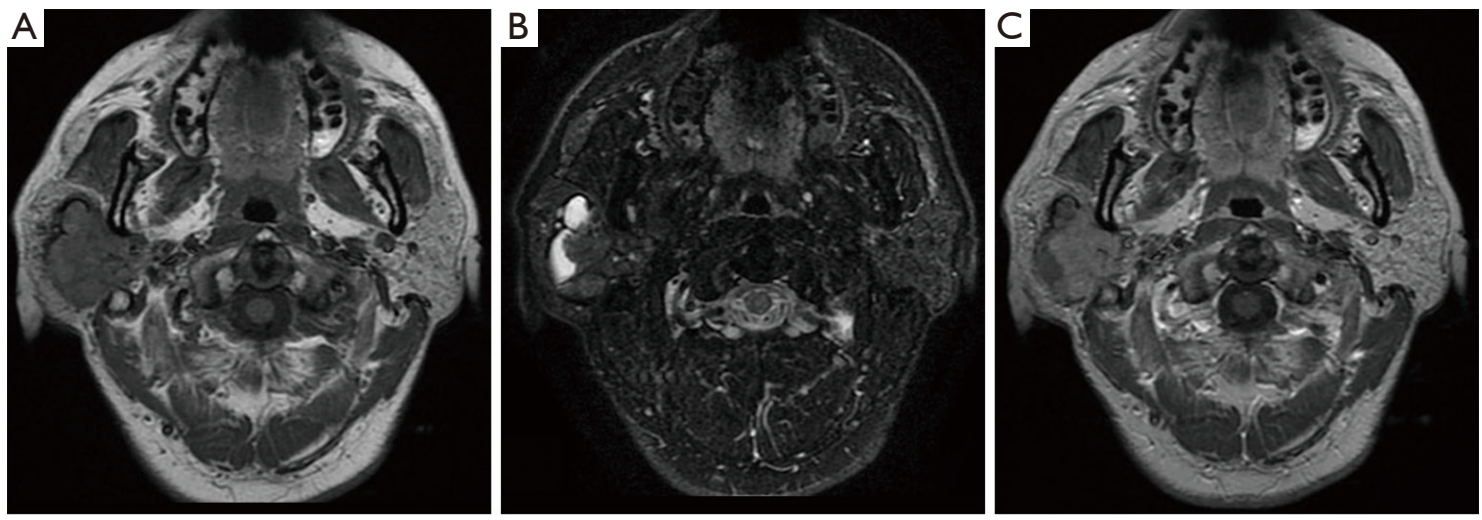

Figure 2 A 47-year-old male with right PGO. (A) T1WI showed an intermediate signal mass with clear boundary and an irregular shape in the right parotid gland; (B) T2WI showed a mixed signal mass; (C) contrast enhanced MRI showed hyperenhancement of the solid part of the mass, and no enhancement of the cystic part. PGO, parotid gland oncocytoma; T1WI, T1 weighted imaging; T2WI, T2 weighted imaging; MRI, magnetic resonance imaging.

\section{Discussion}

Oncocytoma comprises epithelial cells that are rich in mitochondria, named as oncocytes. Oncocytes exist in various forms, from hyperplasia to malignant lesions. However, the presence of abundant oncocytes in the whole lesion is a sign of oncocytoma (5). Oncocytoma can occur in any salivary gland but are more common in the parotid gland. Parotid gland oncocytoma (PGO) is most prevalent among those aged between 60 and 80 years, and does not exhibit any obvious gender tendency (6). The etiology of oncocytoma is unclear. Most of the tumors are benign and exhibit a slow growth. The clinical symptoms are not specific, with most patients presenting with local masses and some with pain. Moreover, the course of the disease can be as long as several years (5). The clinical features of PGO patients in this study are consistent with these findings.

Imaging features of the PGO in this study and those reported in previous studies (5,7-9) are as follows: PGOs are mostly a unilateral, single, well-defined, irregular masses without obvious calcification, some may contain cystic components. The diameter of the lesion rarely exceeds $5 \mathrm{~cm}$. Additional imaging features are: (I) the precontrast US appearance is non-specific and exhibits a well-defined hypoechoic mass. CDFI commonly shows abundant blood flow signals. CEUS often showed hyperenhancement. The size of some lesions on CEUS can be slightly larger than precontrast US. This may be caused by the vascularity and growth pattern. (II) CT showed round or oval, soft tissue masses. A small number of large tumors located in the deep lobe of the parotid gland may extend to the parapharyngeal space through the styloid process mandibular space. The outline of the tumors was squeezed and distorted by the surrounding anatomy, showing a deformed tumor that had not invaded the adjacent tissue structure (8). On postcontrast CT scan, the tumor showed early enhancement and hyperenhancement, which associated with the numerous tumor cells, close arrangement and more blood vessels in the stroma. The enhancement intensity was decreased in the delayed phase. Another feature of the tumors was low-density cleft like lesions (9). (III) MRI signal intensity was variable and influenced by tumor components. T1WI often showed iso/ hypo-intensity, T2WI commonly exhibits iso to hyperintensity. There were also reports of hypointensity on T2WI, which is attributed to the high cellularity and low free water content of oncocytoma (8). Most cases in this study showed homogeneous hyperenhancement. Few cases showed hypoenhancement, which may be due to the lack of vascular components in the tumor stroma. However, Patel et al. (6) also reported the mass as isointense to the native parotid gland, named as "vanishing" on T1weighted postgadolinium sequence, which is associated with the subcellular structure of normal parotid tissue and oncocytes. The cystic components within the tumor showed non-enhanced appearance on contrast enhanced US/CT/MRI.

In the differential diagnosis of PGO, due to overlaps of imaging findings, PGO should be differentiated from pleomorphic adenomas (PA), Warthin tumor, and 


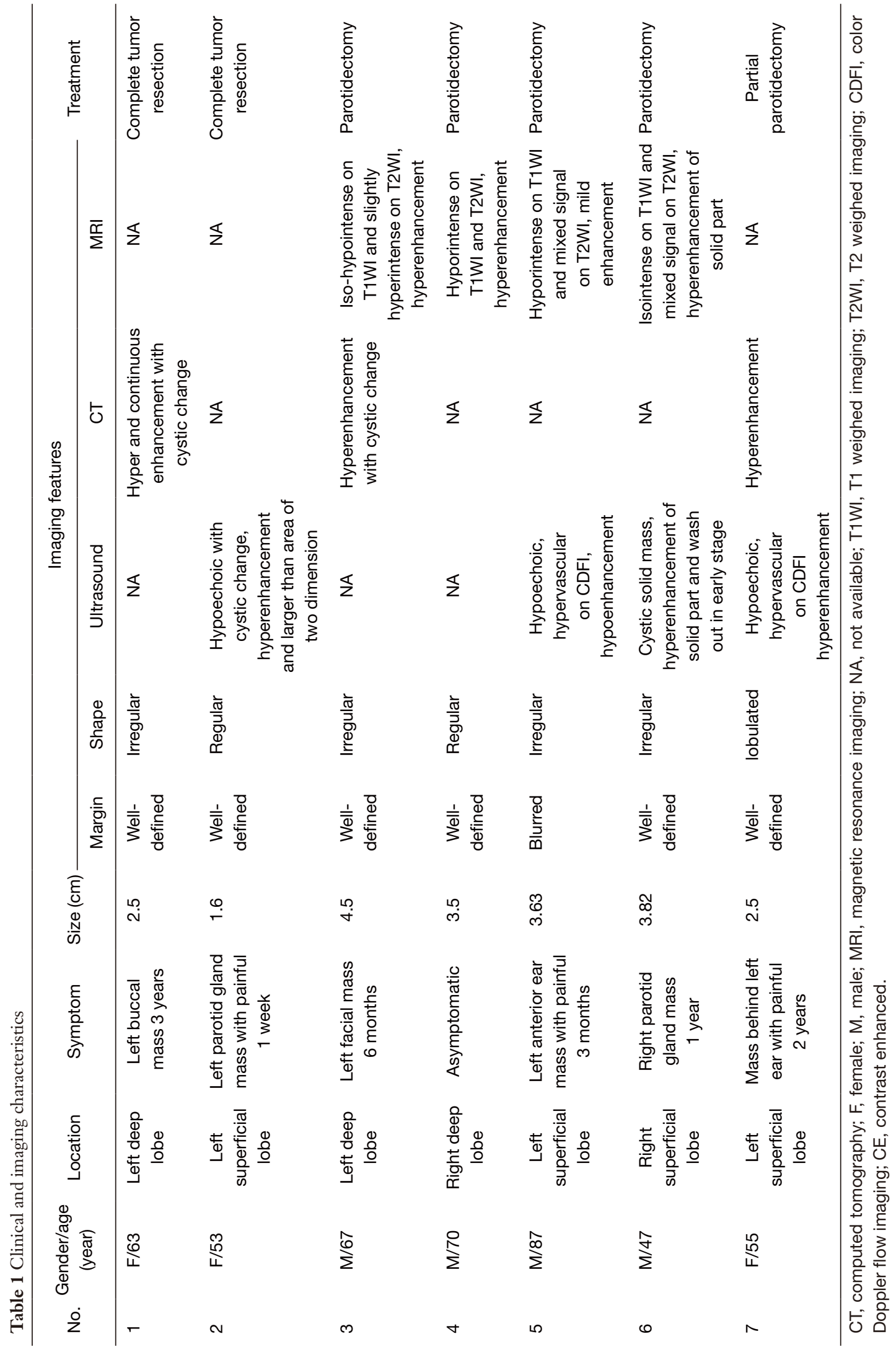


lymphoma. (I) PA are more common in women and some exhibit calcification (10). PA showed mild enhancement in the early stage, which gradually became more enhanced in the delayed phase. However, the PGO showed hyperenhancement in the early stage, and the degree of enhancement was significantly higher than that of PA (11). Moreover, the ADC of PA on DWI was significantly higher than in PGO (12). (II) Warthin tumors are common among males and are localized in the posterior lower quadrant of the superficial parotid gland. Smoking is one of the main risk factors for Warthin tumors. These tumors are likely to undergo cystic transformation, mostly with mild to moderate enhancement. Kato et al. (7) documented that signal intensity ratios (SIRs) of Warthin tumors on T2WI and DWI are higher than those of PGO. In addition, the ADC of Warthin tumors has been shown to be lower than that of PGO. (III) Lymphoma is more common in middleaged and elderly people, often accompanied by swollen lymph nodes in the neck, which are absent in PGO. CT commonly manifests as single or multiple parotid masses with clear margins and necrotic areas. T1WI shows hypointensity, T2WI ranges from hypo- to hyper-intensity. Enhanced CT scans are mild to moderate enhancement and enhanced MRI often reveals hypoenhancement (13).

Surgical resection is the first line therapeutic option for PGO. This method has been documented to have a good prognosis; radiotherapy and chemotherapy are not recommended because of the low sensitivity of oncocytes to chemoradiotherapy (9). Due to histological similarity to benign oncocytoma, malignant oncocytoma may not be correctly diagnosed. Therefore, although malignant transformation and local recurrence of $\mathrm{PGO}$ are not common, clinical follow-up is important (14).

The limitations of this study include: (I) The small sample sizes obtained from a single center, studies with bigger sample sizes and from multiple centers should be conducted to reduce bias, and (II) Image evaluation and ultrasonic image acquisition present some inherent subjectivity. More imaging data of PGOs are needed to allow comparisons with other parotid tumors (benign and malignant).

\section{Conclusions}

PGO lesions in middle-aged and elderly patients usually occur as solid soft tissue masses with clear boundaries, irregular shapes, occasional cystic transformation, and hyperenhancement after contrast enhanced scan. Although imaging can be used for PGO diagnosis, pathological and immunohistochemical analyses remain the gold standard diagnostic methods.

\section{Acknowledgments}

We thank Freescience Editorial Team for technical support. Funding: This work was supported by the Clinical Medicine Research Pilot Project of Shanghai Medical College of Fudan University (grant numbers DGF501022/015), Shanghai Municipal Commission of Science and Technology (grant numbers 19411951200) and Shanghai Hospital Development Center (grant numbers SHDC2020CR3020A).

\section{Footnote}

Reporting Checklist: The authors have completed the MDAR and AME Case Series reporting checklists. Available at http://dx.doi.org/10.21037/gs-20-790

Data Sharing Statement: Available at http://dx.doi. org/10.21037/gs-20-790

Conflicts of Interest: All authors have completed the ICMJE uniform disclosure form (available at http://dx.doi. org/10.21037/gs-20-790). The authors have no conflicts of interest to declare.

Ethical Statement: The authors are accountable for all aspects of the work in ensuring that questions related to the accuracy or integrity of any part of the work are appropriately investigated and resolved. The study was conducted in accordance with the Declaration of Helsinki (as revised in 2013). The study was approved by ethics committee of Huashan hospital, Fudan University (No. KY2013-332) and informed consent was waived for the retrospective nature and all identity information has been removed.

Open Access Statement: This is an Open Access article distributed in accordance with the Creative Commons Attribution-NonCommercial-NoDerivs 4.0 International License (CC BY-NC-ND 4.0), which permits the noncommercial replication and distribution of the article with the strict proviso that no changes or edits are made and the original work is properly cited (including links to both the formal publication through the relevant DOI and the license). See: https://creativecommons.org/licenses/by-nc$\mathrm{nd} / 4.0 \%$. 


\section{References}

1. Iida E, Wiggins RH 3rd, Anzai Y. Bilateral parotid oncocytoma with sponta-neous intratumoral hemorrhage: a rare hypervascular parotid tumor with ASL perfusion. Clin Imaging 2016;40:357-60.

2. Sepúlveda I, Platín E, Spencer ML, et al. Oncocytoma of the parotid gland: a case report and review of the literature. Case Rep Oncol 2014;7:109-16.

3. Sakai E, Yoda T, Shimamoto H, et al. Pathologic and imaging findings of an oncocytoma in the deep lobe of the left parotid gland. Int J Oral Maxillofac Surg 2003;32:563-5.

4. Hamada S, Fujiwara K, Hatakeyama H, et al. Oncocytoma of the Parotid Gland with Facial Nerve Paralysis. Case Rep Otolaryngol 2018;2018:7687951.

5. Popovski V, Benedetti A, Monevska DP, et al. Oncocytoma of the Deep Lobe of the Parotid Gland. Open Access Maced J Med Sci 2016;4:290-2.

6. Patel ND, van Zante A, Eisele DW, et al. Oncocytoma: the vanishing parotid mass. AJNR Am J Neuroradiol 2011;32:1703-6.

7. Kato H, Fujimoto K, Matsuo M, et al. Usefulness of diffusion-weighted MR imaging for differentiating between Warthin's tumor and oncocytoma of the parotid gland. Jpn

Cite this article as: $\mathrm{Lv} \mathrm{K}$, Cao X, Geng D, Zhang J. Imaging features of parotid gland oncocytoma: a case series study. Gland Surg 2021;10(3):870-876. doi: 10.21037/gs-20-790
J Radiol 2017;35:78-85.

8. Shellenberger TD, Williams MD, Clayman GL, et al. Parotid gland oncocytosis: CT findings with histopathologic correlation. AJNR Am J Neuroradiol 2008;29:734-6.

9. Tan TJ, Tan TY. CT features of parotid gland oncocytomas: a study of 10 cases and literature review. AJNR Am J Neuroradiol 2010;31:1413-7.

10. Lee YY, Wong KT, King AD, et al. Imaging of salivary gland tumours. Eur J Radiol 2008;66:419-36.

11. Kakimoto N, Gamoh S, Tamaki J, et al. CT and MR images of pleomorphic ad-enoma in major and minor salivary glands. Eur J Radiol 2009;69:464-72.

12. Habermann CR, Arndt C, Graessner J, et al. Diffusionweighted echo-planar MR imaging of primary parotid gland tumors: is a prediction of different histologic subtypes possible? AJNR Am J Neuroradiol 2009;30:591-6.

13. Zhu L, Wang P, Yang J, et al. Non-Hodgkin lymphoma involving the parotid gland: $\mathrm{CT}$ and $\mathrm{MR}$ imaging findings. Dentomaxillofac Radiol 2013;42:20130046.

14. Sakthikumar KR, Mohanty S, Dineshkumar K. Solitary oncocytoma of the submandibular salivary gland in an adolescent female: A case report. Indian J Otolaryngol Head Neck Surg 2007;59:171-3. 